\title{
A VARIABILIDADE NO DESENVOLVIMENTO E NO ATRITO LINGUÍSTICO: O PAPEL DA EXPERIÊNCIA INDIVIDUAL NO ESTUDO SOBRE OS PADRÕES DE VOT EM SISTEMAS MULTILÍNGUES
}

\section{VARIABILITY IN LANGUAGE DEVELOPMENT AND ATTRITION: THE ROLE OF INDIVIDUAL EXPERIENCE IN A STUDY ON VOT PATTERNS IN MULTILINGUAL SYSTEMS}

\author{
Laura Castilhos Schereschewsky ${ }^{60}$ \\ Ubiratã Kickhöfel Alves ${ }^{61}$
}

\begin{abstract}
RESUMO: Neste estudo, investigamos as variáveis individuais de trajetória do desenvolvimento linguístico de participantes brasileiros bilíngues (Português-L1 e Inglês-L2) e trilíngues (Português-L1, Inglês-L2 e AlemãoL3) e suas possíveis relações com a ocorrência de atrito linguístico do Português-L1 no que refere às mudanças estruturais do sistema, com o desenvolvimento do padrão de Voice Onset Time (VOT) das línguas adicionais. Análises estatísticas de grupo foram conduzidas em conjunto com uma análise individual, para investigar a variabilidade nos dados de VOT e a trajetória linguística dos participantes. Os resultados indicam uma relevante relação entre o desenvolvimento e o uso de línguas adicionais e as mudanças estruturais na L1, bem como uma relação entre o uso e desenvolvimento das duas línguas adicionais.
\end{abstract}

PALAVRAS-CHAVE: Sistemas Dinâmicos Complexos. Variabilidade. Desenvolvimento Linguístico. Atrito Linguístico. Voice Onset Time.

\begin{abstract}
In this study, we investigate possible individual variables in the linguage development of Brazilian bilingual (Portuguese-L1 and English-L2) and trilingual (Portuguese-L1, English-L2 and German-L3) participants, as well as their likely relation with the occurrence of language attrition in Portuguese (L1) in terms of structural changes in the system, with the development of Voice Onset Time (VOT) patterns in the additional languages. Group statistical analyses were conducted along with an individual analysis in order to investigate the variability in VOT and language history data. The results suggest a strong relation between the development and use of additional languages and the structural changes in the L1, as well as a relation between the use and the development of the two additional languages.
\end{abstract}

KEYWORDS: Complex Dynamic Systems. Variability. Language Development. Language Attrition. Voice Onset Time.

\section{Introdução}

Quando Grosjean (1989) sugeriu a inevitabilidade da "mistura" das línguas de um falante bilíngue, implicou-se que esses falantes não poderiam simplesmente alternar entre os sistemas fonético-fonológicos de maneira tão clara, simplesmente porque um sistema linguístico de um falante bilíngue não funciona como a soma de dois sistemas monolíngues. Os subsistemas das duas línguas, na verdade, estariam em constante interação, criando as mais variadas combinações de línguas e interagindo das mais variadas maneiras, num continuum de fala entre os modos monolíngue e bilíngue (GROSJEAN; LI, 2006; GROSJEAN, 1989; 1995; 2013). Não é de se surpreender, então, que cada falante tenha um sistema linguístico caracterizado por trajetórias desenvolvimentais únicas, o que caracteriza uma proposição bastante alinhada à visão de Língua como Sistema Dinâmico Complexo (SDC) $^{62}$ (LARSEN-FREEMAN; CAMERON, 2008; DE BOT; LOWIE; VERSPOOR, 2007,

\footnotetext{
60 Mestranda em Psicolinguística pela Universidade Federal do Rio Grande do Sul. E-mail: castilhoslaura@gmail.com

${ }^{61}$ Professor do Departamento de Línguas Modernas e do Programa de Pós-Graduação em Letras da Universidade Federal do Rio Grande do Sul / Pesquisador do CNPq. E-mail: ukalves@gmail.com

62 Neste artigo, usamos o termo 'Sistema Dinâmico Complexo' (CDS) em vez de 'Sistema Adaptativo Complexo' ou 'Sistema Dinâmico', seguindo De Bot (2017), que concilia com tal termo as premissas da Teoria da Complexidade (LARSEN-FREEMAN, 2015) e dos Sistemas Adaptativos Complexos (LARSEN-FREEMAN; CAMERON, 2008; BECKNER et al., 2009) com a Teoria dos Sistemas Dinâmicos (De BOT et al., 2007).
} 
2011; DE BOT; LOWIE; VERSPOOR, 2013; BECKNER et al., 2009; DE BOT, 2017, entre outros). De acordo com essa visão, a língua está sempre sujeita a mudanças ao longo do tempo e consiste em diversos agentes interagindo uns com os outros o tempo todo. A língua também é tida como adaptativa porque, à medida que os subsistemas interagem, seus agentes modificam o sistema linguístico maior, criando novas interações, de modo que a L1 e a L2 possam mudar constantemente devido às experiências do falante, o que caracteriza a capacidade complexa e dinâmica de auto-organização da língua.

Quando se trata de modelos perceptuais de segunda língua, autores como Flege (1995, 2003, 2007), Best (2001), Best e Tyler (2007) e Perozzo (2017) sugerem que os falantes bilíngues não podem separar as categorias fonético-fonológicas de suas L1 e L2, pois elas existem no mesmo espaço fonético-fonológico, ou seja, elas coexistem e são mutuamente influenciadas no mesmo plano. Na mesma linha, a concepção de língua como um Sistema Dinâmico Complexo, sustentada por estudos como os de Herdina e Jessner (2002), Beckner et al. (2009), De Bot et al. (2013), Kupske (2016) e Pereyron (2017), trazem evidências de que as diferentes trajetórias linguísticas dos falantes podem afetar o seu sistema fonéticofonológico. Dessa forma, atenta-se a uma necessidade de se avaliarem aspectos da experiência linguística dos participantes, fornecendo informações mais detalhadas sobre o histórico de desenvolvimento linguístico para uma compreensão mais próxima da complexidade da experiência bilíngue ( $c f$. GROSJEAN, 2013; ANDERSON et al., 2017, SURRAIN; LUK, 2017; FINGER; ORTIZ-PREUSS, 2018; entre outros).

Em relação às mudanças no sistema, Schmid e de Leeuw (2018) observam que foram necessários anos de pesquisa para que os linguistas desconsiderassem as alterações na L1, como o atrito linguístico, como algo negativo. Schmid e Köpke (2017) definem o atrito linguístico como "fenômenos que surgem na língua nativa de um bilíngue sequencial como consequência da coativação de línguas, transferência ou desuso, em qualquer estágio de desenvolvimento e uso da L2" (SCHMID; KÖPKE, 2017, p. 2). No entanto, de Leeuw (2017) observa que a definição de 'bilíngue' das autoras é estendida a todos os tipos de bilíngues, como bilíngues precoces, tardios, e até os que desenvolveram os dois ou mais idiomas simultaneamente. De acordo com de Leeuw (op. cit.), uma definição mais refinada de atrito linguístico abarcaria "mudanças estruturais na L1 de um bilíngue sequencial tardio, desde que a aquisição estável da L1 preceda a aquisição da L2" (p. 4). A observação de de Leeuw é importante em razão da existência de outras áreas de pesquisa sobre atrito, como o atrito de L1 em crianças que migram para um ambiente de L2 dominante, por exemplo. Nesse caso, alguns autores caracterizariam as mudanças na L1 como resultado do desenvolvimento incompleto da língua materna (PARK, 2018).

No que diz respeito especificamente às mudanças provocadas pela adição de uma nova língua, isto é, à influência interlinguística entre L1 e L2, de Leeuw (2018) verifica que o subsistema fonético-fonológico da L1 está sujeito a mudanças devido à influência de uma língua adicional, mesmo se essa começa a se desenvolver depois da vida adulta. Por exemplo, a autora revisita o estudo de Flege (1987) com dois grupos de bilíngues adultos de inglêsfrancês e francês-inglês, que tiveram suas categorias acústicas "assimiladas" depois de serem imersos em um ambiente de L2-dominante. Embora esse estudo nunca fale em "atrito linguístico", o fenômeno observado dentro dos grupos corresponde à definição de de Leeuw para o termo. Por fim, a autora reafirma a importância de se observar a variabilidade intersujeitos nas análises dos resultados, mesmo que seja possível apontar certas características comuns e padrões de mudança dentro de um grupo.

A fim de ilustrar a importância da variabilidade, podemos usar a proposição de Schmid e Köpke (2017) de que todo e qualquer bilíngue sofre atrito de L1. Segundo as autoras, o processo de atrito linguístico começa logo no início do desenvolvimento da L2, exatamente porque, do ponto de vista dinâmico, não podemos separar uma língua da outra, 
uma vez que a coativação pode afetar o processamento ou a produção da língua em uso. Novamente, a importância do fator 'tempo' deve ser enfatizada porque, à medida em que o tempo passa, essa interação pode eventualmente levar a mudanças ou reestruturações dos subsistemas como resultado da influência mútua entre as línguas. Além disso, Schmid e Leeuw (2018) propõem que os efeitos desse fenômeno podem ser observados da L1 para a L2 e da L2 para a L1 em um continuum onde o desenvolvimento é gradual, mas não necessariamente linear. Ao se adotar essa ideia e, portanto, aceitar que não há maneira certa de estabelecer dois pontos diferentes neste continuum do desenvolvimento, a proposição de que todo bilíngue está atritado se torna mais tangível, e a necessidade de considerar a variabilidade entre sujeitos torna-se ainda mais sustentável.

Essa necessidade metodológica está em consonância com a visão de variabilidade, defendida por Lowie e Verspoor (2015), como sendo o principal aspecto a ser analisado em estudos sobre o desenvolvimento linguístico. Segundo os autores, do ponto de vista de língua como um SDC, o desenvolvimento de uma língua não é algo a ser alcançado e finalizado. É, por sua vez, um processo. Nesse sentido, uma vez que cada indivíduo possui características muito particulares, cada processo é único, daí o motivo da importância da variabilidade. Além disso, os autores sugerem que a própria existência da variação individual é uma força propulsora para a mudança, devido à capacidade intrínseca de auto-organização do sistema. Por outro lado, reconhecem o fato de que grupos de indivíduos cuidadosamente selecionados podem apresentar padrões de desenvolvimento e até parecerem semelhantes ou homogêneos, mas que cada pessoa ainda tem sua própria história e, portanto, os estudos devem considerar análises individuais e de grupo como complementares (LOWIE; VERSPOOR, 2019).

Partindo das caracterizações acima, este estudo tem o objetivo de investigar a relação de variáveis da trajetória linguística com mudanças estruturais nas categorias acústicas de participantes multilíngues, concentrando-se em mudanças que apontam a um possível atrito linguístico. Para isso, são analisadas variáveis sobre a trajetória e a experiência linguística, obtidas através do preenchimento de questionários por participantes bilíngues (PB e inglês) e trilíngues ( $\mathrm{PB}$, inglês e alemão), de modo a se investigarem possíveis correlações com valores da produção de Voice Onset Time $\left(\mathrm{VOT}^{63}\right)$, além de possíveis correlações entre as próprias produções nas diferentes línguas. Diferentemente do $\mathrm{PB}$, as palavras iniciadas por $/ \mathrm{p} /, / \mathrm{t} / \mathrm{e} / \mathrm{k} /$ em inglês e alemão são aspiradas (produzidas com um VOT positivo), e por isso espera-se que, com o desenvolvimento das línguas adicionais, os falantes multilíngues apresentem mudanças estruturais no sistema de L1, produzindo um VOT mais longo nessas consoantes, dada a intrínseca interconectividade das línguas.

\section{Metodologia}

Participaram deste estudo 20 voluntários, divididos em dois grupos, um com 10 bilíngues (Grupo A) e um com 10 trilíngues (Grupo B) ${ }^{64}$. O Grupo A era formado por 10 falantes nativos do Português, nascidos e residentes em Porto Alegre (RS), aprendizes de Inglês como L2 (média de idade 24,40 anos, DP 3,8). Todos os participantes bilíngues

\footnotetext{
${ }^{63}$ Voice Onset Time refere-se ao intervalo de tempo entre a explosão de uma consoante plosiva e o início da vibração vocálica do segmento seguinte (LISKER; ABRAMSON, 1964). Em plosivas iniciais, considera-se que o Português tenha um padrão de VOT Zero, pois esse intervalo é de curta duração, enquanto o Inglês e o Alemão têm VOT positivo, com um longo intervalo de tempo. Em Abramson \& Whalen (2017), encontra-se uma longa discussão sobre os 50 anos de estudos sobre VOT.

${ }^{64}$ Os dados do presente trabalho fazem parte de um estudo maior, apresentado em Schereschewsky (2018), referente ao atrito linguístico no Português (L1) por falantes de Inglês (L2) e Alemão (L3). No estudo em questão, contamos também com os dados de 20 monolíngues (10 de PB e 10 de Inglês), coletados e apresentados em Kupske (2016), para fins de controle.
} 
possuíam o ensino médio completo e cursavam o sétimo semestre do ensino superior em Letras-Inglês pela universidade dos autores no período da coleta de dados. O Grupo B era formado por 10 falantes nativos do Português, também nascidos e residentes em Porto Alegre (RS), aprendizes de Inglês como L2 e de Alemão como L3 (média de idade 27,90 anos, DP 9,8). Desses, um possuía o ensino superior completo; sete estavam cursando o ensino superior; um possuía pós graduação; e um era pós-graduando.

Após a leitura e assinatura do Termo de Consentimento Livre e Esclarecido, os participantes foram submetidos ao Questionário de Histórico da Linguagem de Scholl e Finger (2013), que mapeou o histórico linguístico e sua vivência com as línguas de que tinham conhecimento. A literatura estudada por Scholl (2016) apontou congruências e uma relativa correlação entre os dados obtidos no questionário, como a autoavaliação da proficiência nas línguas estrangeiras e a experiência linguística, e o desempenho associado a testes de proficiência tradicionais desses bilíngues. Como o questionário cobria muitos aspectos sobre as experiências linguísticas diárias dos falantes, foram selecionadas apenas as questões mais pertinentes a um estudo sobre atrito linguístico no sistema fonético-fonológico. As variáveis estudadas foram 'Língua 1'; 'Língua 2'; 'Língua 3'; 'Idade'; 'Idade em que começou a aprender (x) língua'; 'Idade em que começou a utilizar ativamente (x) língua'; 'Idade em que tornou-se fluente em (x) língua'; 'Anos de estudo de (x) língua'; 'Número de meses em país em que a língua é falada'; 'Número de meses em escola / trabalho em que a língua é falada'; 'Percentagem do tempo em que o participante usa cada língua diariamente'; 'Soma do número de horas em que se fala, assiste TV, ouve música e joga videogame em cada língua'; 'Autoavaliação da compreensão auditiva (de 1 a 6)'; e 'Autoavaliação da fala (de 1 a 6)'. Além disso, como fator complementar para critério de exclusão do estudo, todos os participantes teriam de previamente obter pontuação mínima de 65 na versão em inglês do teste de proficiência $C$-Test $t^{65}$ e 50 na versão em alemão do referido teste.

Por fim, os dois grupos experimentais realizaram uma tarefa de produção para a coleta dos dados acústicos. A tarefa consistia na leitura em voz alta de slides com 23 frases-veículo "Eu diria _.", em PB, "I would say _.", em inglês, e "Ich sage_.", em alemão, seguidas por palavras-alvo com /p/, /t/ e /k/ em posição inicial, todas com três repetições apresentadas em ordem aleatória, além de frases com cinco palavras distratoras. Mantivemos a mesma metodologia previamente empregada em Kupske (2016) e Schereschewsky (2018), em que cada grupo realizou uma tarefa de leitura para cada língua de seu conhecimento, em ordem de aquisição, pois os resultados desses estudos serviram de base para os nossos Grupos de Controle. As coletas dos dados acústicos ocorreram em ambiente silencioso, através de um microfone gravador Zoom $\mathrm{H} 2$, e armazenados com identificação apenas do número do participante. Analisamos esses dados acusticamente no software Praat - v. 6.0.40 (BOERSMA; WEENINK, 2018), seguindo os critérios dois dois estudos supracitados (selecionando o período de surdez da consoante inicial imediatamente após a soltura da plosiva até a marca do primeiro pulso regular da vibração vocálica seguinte), e os valores de tais medições foram correlacionados com os dados da análise de histórico linguístico, quantificados numericamente e inseridos em tabelas.

\section{Resultados e Discussão}

Para atendermos os objetivos descritos na Introdução deste artigo, serão discutidos aqui os resultados estatísticos para as correlações entre as variáveis de trajetória linguística e os valores descritos de VOT. Serão apresentadas as correlações entre as médias de VOT das

\footnotetext{
${ }^{65}$ Teste do tipo de redundância reduzida, que consiste no preenchimento de lacunas usado como medida para testar proficiência generalizada em línguas adicionais ( $c f$. RAATZ; KLEIN-BRALEY, 2002).
} 
diferentes línguas, em seguida as correlações entre essas médias e as particularidades apontadas nos questionários de vivência linguística. Por fim, objetiva-se explorar esses dados e fazer uma análise qualitativa mais individual dessas produções, observando as relações entre as médias de VOT e algumas características particulares de cada participante, comparando com os dados das médias dos participantes monolíngues de Kupske (2016), tomados como controle de padrão monolíngue. Os dados dos participantes encontram-se nas tabelas a seguir, divididos entre dados dos participantes bilíngues do Grupo A (Tabela 1) e dos participantes trilíngues do Grupo B (Tabela 2).

Tabela 1 - Dados das médias de VOT e trajetória linguística do Grupo A

\begin{tabular}{|c|c|c|c|c|c|c|c|c|c|c|}
\hline Grupo & 3 & 3 & 3 & 3 & 3 & 3 & 3 & 3 & 3 & 3 \\
\hline Participante & 21 & 22 & 23 & 24 & 25 & 26 & 27 & 28 & 29 & 30 \\
\hline p_port & 26,91 & 31,42 & 14,67 & 26,13 & 41,19 & 24,09 & 23,47 & 30,46 & 30,37 & 26,72 \\
\hline p_ing & 51,38 & 36,11 & 22,13 & 47,75 & 49,01 & 32,44 & 22,00 & 26,23 & 79,84 & 33,34 \\
\hline p_ale & - & - & - & - & - & - & - & - & - & - \\
\hline t_port & 44,78 & 33,07 & 25,14 & 30,36 & 35,71 & 25,74 & 28,45 & 27,57 & 28,32 & 31,03 \\
\hline t_ing & 81,16 & 41,81 & 59,82 & 61,57 & 95,49 & 39,06 & 38,48 & 39,87 & 99,56 & 54,56 \\
\hline t_ale & - & - & - & - & - & - & - & - & - & - \\
\hline k_port & 86,96 & 65,68 & 56,59 & 56,53 & 79,47 & 45,30 & 56,71 & 61,20 & 67,41 & 52,22 \\
\hline $\mathbf{k}$ ing & 95,59 & 64,58 & 61,97 & 84,36 & 92,69 & 77,87 & 62,08 & 85,69 & 104,34 & 59,55 \\
\hline k_ale & - & - & - & - & - & - & - & - & - & - \\
\hline C-Test ing & 80,5 & 85,5 & 75 & 81 & 78 & 67 & 88,5 & 72 & 87 & 70 \\
\hline C-Test ale & - & - & - & - & - & - & - & - & - & - \\
\hline Idade & 21 & 28 & 21 & 31 & 24 & 21 & 21 & 28 & 22 & 27 \\
\hline começou a aprender_ing & 10 & 1 & 9 & 6 & 17 & 13 & 8 & 10 & 3 & 10 \\
\hline utilizar ativamente_ing & 12 & 10 & 11 & 10 & 19 & 16 & 11 & 14 & 14 & 15 \\
\hline fluente_ing & 15 & 18 & 12 & 22 & 20 & 17 & 16 & $?$ & 17 & 18 \\
\hline anos estudo_ing & 11 & 27 & 12 & 25 & 7 & 8 & 13 & 18 & 19 & 17 \\
\hline meses país_ing & 3 & 0 & 5 & 2 & 0 & 0 & 1 & 1 & 0 & 0 \\
\hline meses escola/trabalho_ing & 1 & 6 & 25 & 96 & 96 & 36 & 0 & 38 & 156 & 36 \\
\hline \% tempo uso língua_ing & 24,5 & 25 & 20 & 30 & 15 & 20 & 10 & 30 & 35 & 20 \\
\hline Soma $n^{a}$ horas uso_ing & 7 & 7 & 7 & 7,5 & 14 & 9 & 4 & 5 & 13 & 4 \\
\hline Autoaval. audit_ing & 6 & 6 & 6 & 6 & 5 & 4 & 5 & 4 & 6 & 6 \\
\hline Autoavaliação fala_ing & 5 & 6 & 6 & 6 & 5 & 3 & 4 & 3 & 6 & 5 \\
\hline Tradução & $\operatorname{sim}$ & $\operatorname{sim}$ & - & - & - & - & - & - & $\operatorname{sim}$ & - \\
\hline
\end{tabular}


Volume 14 - Número 1 - mai/ago de 2019

Tabela 2 - Dados das médias de VOT e trajetória linguística do Grupo B

\begin{tabular}{|c|c|c|c|c|c|c|c|c|c|c|}
\hline Grupo & 4 & 4 & 4 & 4 & 4 & 4 & 4 & 4 & 4 & 4 \\
\hline Participante & 31 & 32 & 33 & 34 & 35 & 36 & 37 & 38 & 39 & 40 \\
\hline p_port & 24,50 & 26,62 & 21,82 & 22,98 & 24,15 & 30,44 & 26,85 & 37,64 & 31,04 & 24,46 \\
\hline p_ing & 44,42 & 40,89 & 39,10 & 37,37 & 37,33 & 37,53 & 49,30 & 46,67 & 41,44 & 43,17 \\
\hline p_ale & 41,23 & 46,11 & 27,91 & 34,95 & 29,78 & 40,03 & 48,00 & 41,84 & 31,08 & 39,39 \\
\hline t_port & 31,62 & 30,84 & 24,66 & 30,47 & 30,48 & 37,39 & 34,30 & 39,43 & 32,40 & 40,74 \\
\hline t_ing & 52,14 & 80,52 & 55,67 & 60,30 & 81,91 & 42,97 & 92,60 & 56,52 & 57,08 & 56,57 \\
\hline t_ale & 45,12 & 84,86 & 39,15 & 52,76 & 66,89 & 51,90 & 73,31 & 47,07 & 42,89 & 47,75 \\
\hline k_port & 63,32 & 60,92 & 58,70 & 66,37 & 55,13 & 67,11 & 64,39 & 62,05 & 60,11 & 78,21 \\
\hline k_ing & 87,49 & 92,45 & 70,74 & 91,27 & 74,65 & 66,43 & 81,01 & 84,72 & 70,58 & 79,25 \\
\hline k_ale & 63,40 & 99,32 & 60,46 & 91,87 & 67,60 & 73,09 & 81,01 & 75,41 & 63,58 & 77,15 \\
\hline C-Test ing & 80,5 & 65,5 & 81,5 & 67 & 73,5 & 85,5 & 79 & 74 & 65 & 76,5 \\
\hline C-Test ale & 65,5 & 52,5 & 76 & 52 & 53,5 & 57 & 67,5 & 59,5 & 73 & 61 \\
\hline Idade & 24 & 26 & 27 & 21 & 20 & 27 & 21 & 37 & 22 & 54 \\
\hline começou a aprender_ing & 11 & 6 & 10 & 10 & 6 & 5 & 7 & 10 & 4 & 12 \\
\hline utilizar ativamente_ing & 13 & 12 & 13 & 13 & 10 & 11 & 12 & 18 & 12 & 18 \\
\hline fluente_ing & 17 & 16 & 14 & 17 & 14 & 18 & 16 & 18 & 18 & 20 \\
\hline anos estudo_ing & 13 & 20 & 17 & 11 & 14 & 22 & 14 & 27 & 18 & 42 \\
\hline meses país_ing & 1 & 0 & 0 & 1 & 0 & 0 & 0 & 0 & 0 & 2 \\
\hline meses escola/trabalho_ing & 36 & 18 & 84 & 0 & 0 & 6 & 0 & 0 & 18 & 80 \\
\hline \% tempo uso língua_ing & 15 & 10 & 10 & 7 & 10 & 25 & 17 & 10 & 10 & 30 \\
\hline Soma $\mathrm{n}^{\mathrm{a}}$ horas uso_ing & 4,5 & 3,5 & 2,3 & 2,7 & 4 & 10,5 & 14 & 3 & 3 & 9 \\
\hline Autoaval. audit_ing & 6 & 6 & 5 & 5 & 6 & 6 & 6 & 5 & 5 & 6 \\
\hline Autoavaliação fala_ing & 6 & 5 & 4 & 6 & 6 & 6 & 4 & 4 & 4 & 6 \\
\hline começou a aprender_ale & 13 & 23 & 11 & 17 & 16 & 10 & 16 & 25 & 17 & 50 \\
\hline utilizar ativamente_ale & 21 & 23 & 15 & 18 & 17 & 14 & 16 & 25 & 19 & 52 \\
\hline fluente_ale & 23 & - & 17 & - & - & - & 20 & - & - & - \\
\hline anos estudo_ale & 11 & 3 & 16 & 4 & 4 & 17 & 5 & 12 & 5 & 4 \\
\hline meses país_ale & 3 & 0 & 2 & 0 & 0 & 2 & 2 & 0 & 0 & 1 \\
\hline meses escola/trabalho_ale & 16 & 16 & 88 & 0 & 0 & 6 & 2 & 0 & 32 & 30 \\
\hline \% tempo uso língua_ale & 8 & 10 & 3 & 3 & 5 & 5 & 2 & 10 & 20 & 5 \\
\hline Soma $n^{a}$ horas uso_ale & 2,7 & 2 & 0,2 & 3,6 & 1 & 2,5 & 7 & 2 & 5 & 3 \\
\hline Autoaval. audit_ale & 4 & 5 & 6 & 3 & 5 & 4 & 5 & 4 & 5 & 4 \\
\hline Autoavaliação fala_ale & 5 & 3 & 5 & 5 & 3 & 4 & 5 & 3 & 4 & 3 \\
\hline Tradução & - & $\operatorname{sim}$ & - & - & $\operatorname{sim}$ & - & $\operatorname{sim}$ & - & - & $\operatorname{sim}$ \\
\hline
\end{tabular}


Nas próximas tabelas, apresentaremos as correlações entre as variáveis referentes às médias de produção de VOT das diferentes línguas, e, em seguida, as correlações entre essas médias e as particularidades de cada grupo apontadas nos questionários de vivência linguística.

Tabela 3 - Correlações entre as médias de VOT em PB e Inglês pelo Grupo A

\begin{tabular}{lc}
\hline$/ \mathbf{p} /$ & $\mathrm{r}=0,46, \mathrm{p}=0,090^{* 66}$ \\
$/ \mathbf{t} /$ & $\mathrm{r}=0,45, \mathrm{p}=0,097^{*}$ \\
$/ \mathbf{k} /$ & $\mathrm{r}=0,60, \mathrm{p}=0,034^{* * *}$ \\
\hline
\end{tabular}

Com os dados da Tabela 3, podem-se identificar os resultados das análises estatísticas que correlacionaram as médias de VOT para as três consoantes estudadas entre produções em PB e Inglês, línguas de conhecimento do Grupo A. O coeficiente de correlação de Pearson identificou correlações positivas marginalmente significativas para as produções da plosiva surda bilabial, $r=0,46, p=0,090$, e para a plosiva surda alveolar, $r=0,45, p=0,097$, ao passo que, para a plosiva surda velar, foram encontradas correlações positivas significativas, $r$ $=0,60, p=0,034$. O que esses dados sugerem é que, para o Grupo A, há correlações positivas entre os valores de VOT em Português e Inglês, ou seja, quanto maior a média de produção de VOT em uma das línguas, maior a média na outra. De certa forma, isso já sugere uma alteração no sistema fonético-fonológico desses participantes bilíngues, pois a presença de uma nova língua já demonstra uma mudança em todo o sistema linguístico. Essa discussão, adicionada à discussão sobre o possível atrito linguístico, será retomada adiante. A Tabela 4, a seguir, traz informações sobre o Grupo B.

Tabela 4 - Correlações entre as médias de VOT em PB, Inglês e Alemão pelo Grupo B

\begin{tabular}{ccc}
\hline PB-inglês & /p/ & $\mathrm{r}=0,389, \mathrm{p}=0,266$ \\
& $/ \mathbf{t} /$ & $\mathrm{r}=-0,206, \mathrm{p}=0,569$ \\
$/ \mathbf{k} /$ & $\mathrm{r}=0,180, \mathrm{p}=0,618$ \\
\hline PB-alemão & $/ \mathbf{p} /$ & $\mathrm{r}=0,311, \mathrm{p}=0,382$ \\
& $/ \mathbf{t} /$ & $\mathrm{r}=-0,051, \mathrm{p}=0,889$ \\
& $/ \mathbf{k} /$ & $\mathrm{r}=0,361, \mathrm{p}=0,305$ \\
\hline inglês-alemão & $/ \mathbf{p} /$ & $\mathrm{r}=0,656, \mathrm{p}=0,039 * * *$ \\
& $/ \mathbf{t} /$ & $\mathrm{r}=0,829, \mathrm{p}=0,003 * * *$ \\
& $/ \mathbf{k} /$ & $\mathrm{r}=0,691, \mathrm{p}=0,027 * * *$ \\
\hline
\end{tabular}

Para a Tabela 4, mostrada acima, podemos identificar os resultados das análises estatísticas que correlacionaram, pelo Coeficiente de Correlação de Pearson, as médias de

\footnotetext{
${ }^{66}$ Legenda das tabelas: $* * *=$ significativo; $*=$ marginalmente significativo; sem asteriscos $=$ não significativo.
} 
VOT para cada uma das três consoantes em três blocos, entre produções em PB e Inglês, PB e Alemão, e Inglês e Alemão, todas as línguas de conhecimento do Grupo B. Sobre os dados, não são encontrados resultados significativos para as correlações entre a L1 e as línguas adicionais dos participantes. Essa ausência de correlação entre o PB e o Inglês ou Alemão dos trilíngues está em desacordo com o que era esperado, pois, na possibilidade de atrito linguístico, era de se esperar que uma maior média de VOT em PB estivesse em consonância com uma maior média nas línguas adicionais. É possível que, pela grande variabilidade dos participantes, suas produções ainda não estejam bem estabelecidas. No entanto, concentremonos nas correlações entre a L2 e a L3 dos trilíngues. O coeficiente de correlação de Pearson identificou correlações positivas para as produções da plosiva surda bilabial, $\mathrm{r}=0,656, \mathrm{p}=$ 0,039 , da plosiva surda alveolar, $r=0,829, p=0,003$, e da plosiva surda velar, $r=0,691, p=$ 0,027 . Assim, uma maior média de produção de VOT para essas consoantes em língua inglesa está associada a uma maior média de produção de VOT em língua alemã. Dessa forma, é possível especular que, como são duas línguas tipologicamente próximas, as duas estão se auxiliando mutuamente na produção de um VOT mais longo.

Além disso, como previamente mencionado, também foram analisadas as possíveis correlações entre as médias dos valores de VOT e as variáveis apontadas nos questionários de vivência linguística de Scholl e Finger (2013). Nas tabelas que seguem, são reportadas apenas as correlações que resultaram significativas ou marginalmente significativas, por motivos de limitação de espaço. A Tabela 5 traz os resultados para as correlações entre as variáveis e as médias de VOT para cada consoante entre as duas línguas do Grupo A, realizadas com o Coeficiente de Correlação de Pearson.

Tabela 5 - Correlações significativas encontradas entre as médias de VOT e variáveis de experiência linguística do Grupo A

\begin{tabular}{|c|c|c|}
\hline \multirow[t]{3}{*}{ PB } & $/ \mathbf{p} /$ & $\begin{array}{l}\text { idade utilizar ativamente inglês }(\mathrm{r}=0,57, \mathrm{p}=0,042)^{* * *} \\
\text { idade tornou-se fluente inglês }(\mathrm{r}=0,66, \mathrm{p}=0,027)^{* * *} \\
\text { soma } \mathrm{n}^{\circ} \text { horas uso/dia inglês }(\mathrm{r}=0,543, \mathrm{p}=0,052)^{*}\end{array}$ \\
\hline & $/ \mathbf{t} /$ & N.S. \\
\hline & $/ \mathbf{k} /$ & N.S. \\
\hline \multirow[t]{3}{*}{ Inglês } & $/ \mathbf{p} /$ & $\begin{array}{l}\mathrm{n}^{\mathrm{o}} \text { meses trabalho/escola inglês }(\mathrm{r}=0,78, \mathrm{p}=0,004)^{* * *} \\
\text { percentagem uso inglês }(\mathrm{r}=0,58, \mathrm{p}=0,037)^{* * *} \\
\text { soma } \mathrm{n}^{\circ} \text { de horas uso/dia inglês }(\mathrm{r}=0,72, \mathrm{p}=0,009)^{* * *} \\
\text { autoavaliação fala inglês }(\mathrm{r}=0,46, \mathrm{p}=0,093)^{*}\end{array}$ \\
\hline & $/ \mathbf{t} /$ & $\begin{array}{c}\mathrm{n}^{\mathrm{o}} \text { meses trabalho/escola inglês }(\mathrm{r}=0,656, \mathrm{p}=0,020)^{* * *} \\
\text { soma } \mathrm{n}^{\mathrm{o}} \text { horas uso/dia inglês }(\mathrm{r}=0,77, \mathrm{p}=0,004)^{* * *} \\
\text { autoavaliação fala inglês }(\mathrm{r}=0,51, \mathrm{p}=0,068)^{*}\end{array}$ \\
\hline & $/ \mathbf{k} /$ & $\begin{array}{c}\mathrm{n}^{\mathrm{o}} \text { meses trabalho/escola inglês }(\mathrm{r}=0,656, \mathrm{p}=0,020)^{* * *} \\
\text { percentagem uso inglês }(\mathrm{r}=0,56, \mathrm{p}=0,047)^{* * *} \\
\text { soma } \mathrm{n}^{\circ} \text { horas uso/dia inglês }(\mathrm{r}=0,694, \mathrm{p}=0,013) * * *\end{array}$ \\
\hline
\end{tabular}

Correlações positivas significativas foram encontradas entre algumas variáveis de experiência linguística e um maior valor de produção de VOT. Ainda que não tenham sido encontradas correlações para $/ \mathrm{t} / \mathrm{e} / \mathrm{k} /$ do $\mathrm{PB}$, surpreendentemente foram encontradas 
correlações significativas para $/ \mathrm{p} /$, que deveria ser a plosiva com maior variabilidade entre os falantes. Uma das correlações positivas encontradas, referente à soma do número de horas de uso de Inglês por dia, também está presente nas correlações entre as variáveis e o VOT para as três consoantes do Inglês. No geral, ainda é possível observar que essa é a variável que se mostrou coerente para as três plosivas estudadas do Inglês, ou seja, onde há correlações significativas, parece ser essa a variável mais relevante para a produção de VOT para o grupo bilíngue. Nesse caso, é viável sugerir que, nas produções de /p/ em PB e nas produções das três consoantes em Inglês, um maior valor de VOT está associado a uma maior soma do número de horas de uso de Inglês por dia. A Tabela 6, a seguir, refere-se aos resultados associados ao Grupo B.

Tabela 6 - Correlações significativas encontradas entre as médias de VOT e variáveis de experiência linguística do Grupo B

\begin{tabular}{|c|c|c|}
\hline \multirow[t]{3}{*}{ PB } & $/ \mathbf{p} /$ & N.S. \\
\hline & $/ \mathbf{t} /$ & $\begin{array}{l}\text { idade utilizar ativamente inglês }(\mathrm{r}=0,601, \mathrm{p}=0,066)^{*} \\
\text { idade tornou-se fluente inglês }(\mathrm{r}=0,815, \mathrm{p}=0,003)^{* * *} \\
\text { anos de estudo de inglês }(\mathrm{r}=0,730, \mathrm{p}=0,017)^{* * *} \\
\text { percentagem uso inglês }(\mathrm{r}=0,668, \mathrm{p}=0,035)^{* * *} \\
\text { idade começou aprender alemão }(\mathrm{r}=0,620, \mathrm{p}=0,056)^{*} \\
\text { idade utilizar ativamente alemão }(\mathrm{r}=0,601, \mathrm{p}=0,066)^{*}\end{array}$ \\
\hline & $/ \mathbf{k} /$ & $\begin{array}{c}\text { idade tornou-se fluente inglês }(\mathrm{r}=0,761, \mathrm{p}=0,056)^{*} \\
\text { meses país inglês }(\mathrm{r}=0,607, \mathrm{p}=0,063)^{*} \\
\text { percentagem uso inglês }(\mathrm{r}=0,660, \mathrm{p}=0,038)^{* * *} \\
\text { autoavaliação auditiva alemão }(\mathrm{r}=-0,691, \mathrm{p}=0,027)^{* * *}\end{array}$ \\
\hline \multirow[t]{3}{*}{ Inglês } & $/ \mathbf{p} /$ & soma $n^{\circ}$ horas uso/dia alemão $(r=0,554, p=0,096)^{*}$ \\
\hline & $/ \mathbf{t} /$ & anos de estudos de alemão $(\mathrm{r}=-0,649, \mathrm{p}=0,042)^{* * *}$ \\
\hline & $/ \mathbf{k} /$ & idade tornou-se fluente alemão $(\mathrm{r}=0,992, \mathrm{p}=0,083)^{*}$ \\
\hline \multirow[t]{3}{*}{ Alemão } & $/ \mathbf{p} /$ & soma $\mathrm{n}^{\mathrm{o}}$ horas uso/dia inglês $(\mathrm{r}=0,566, \mathrm{p}=0,088)^{*}$ \\
\hline & $/ \mathbf{t} /$ & autoavaliação auditiva inglês $(\mathrm{r}=0,561, \mathrm{p}=0,092)^{*}$ \\
\hline & $/ \mathbf{k} /$ & nota $\mathrm{C}$-Test alemão $(\mathrm{r}=0,684, \mathrm{p}=0,029)^{* * *}$ \\
\hline
\end{tabular}

A Tabela 6 traz os resultados para as correlações entre as variáveis e as médias de VOT para cada consoante entre as três línguas faladas pelo Grupo B, também avaliadas com o Coeficiente de Correlação de Pearson. Novamente, podem-se observar correlações positivas significativas entre algumas variáveis de experiência linguística e um maior valor de produção de VOT para todas as consoantes, em todas as línguas de conhecimento desses trilíngues, salvo para as produções de /p/ em PB. Alguns dados precisam ser mencionados, como o fato de que a soma de número de horas se mostrou uma variável significativa tanto para a produção de /p/ em Inglês quanto de /p/ em Alemão. Mais interessantes são a soma de horas de uso em Alemão, que se correlacionou significativamente nas produções em Inglês, e a soma de horas de uso de Inglês, nas produções em Alemão. Isso sugere que as duas línguas apresentam uma forte relação, fato esse que pode estar relacionado à influência mútua entre a L2 e a L3, tipologicamente semelhantes. Além disso, a idade em que o participante se tornou fluente em Inglês e a percentagem de uso dessa língua estão correlacionadas com um maior valor de VOT para /t/ e /k/ em PB, indicando, também, uma influência da L2 na produção em 
L1, o que já pode estar dentro da discussão sobre atrito linguístico. Ademais, curiosamente, alguns resultados encontrados foram de correlações negativas significativas, como é o caso de /t/ em Inglês e 'anos de estudos de Alemão'. Dessa forma, é possível especular sobre a influência da L2 sobre a L3 e, nesse caso, um maior valor de VOT está associado a um menor valor de anos de estudo de Alemão. Tal dado é deveras curioso, pois esses resultados indicariam que quanto menos anos se estuda Alemão, mais longa a produção de VOT.

Durante a entrevista, três dos participantes bilíngues e quatro dos participantes trilíngues também afirmaram realizar atividades recorrentes de tradução e/ou interpretação, todos em língua inglesa. Apenas um dos participantes trilíngues também afirmou traduzir para o alemão. Com esses dados, foi possível realizar mais um teste estatístico para correlacionar os valores de VOT e a ocorrência de atividades de tradução/interpretação, com o coeficiente de correlação Ponto-Bisserial. No que concerne ao Grupo A, encontramos correlações positivas marginalmente significativas para a produção de $/ \mathrm{k} / \mathrm{em} \mathrm{PB}, \mathrm{r}=0,578, \mathrm{p}=0,080$, e também marginalmente significativas para o $/ \mathrm{p} / \mathrm{em}$ inglês, $\mathrm{r}=0,615, \mathrm{p}=0,058$. No que concerne ao Grupo B, encontramos correlações positivas significativas para o /t/ em inglês, $r$ $=0,779, \mathrm{p}=0,008$, e também para $\mathrm{o} / \mathrm{t} / \mathrm{em}$ alemão, $\mathrm{r}=0,754, \mathrm{p}=0,012$. Ou seja, para essas consoantes, uma maior produção de VOT parece estar associada ao exercício de atividades de tradução e/ou interpretação, que implicam um contato ainda maior com as línguas adicionais.

No caso do $\mathrm{PB}$, não foram encontradas muitas correlações significativas entre os valores de VOT e os fatores de experiência linguística, como é o caso de /t/ e /k/ para o Grupo A e de /p/ para o Grupo B, o que pode estar relacionado ao fato de haver muita variabilidade nas produções em língua portuguesa, pois esses participantes estão no meio de um processo de atrito linguístico, em que seu sistema inteiro de L1 está em mudança, em consonância com a visão de língua como um sistema dinâmico, adaptativo e complexo. De fato, foram verificadas algumas correlações positivas significativas para essas variáveis e a produção nas línguas adicionais, porém também eram esperadas correlações negativas significativas para as variáveis referentes à idade. No caso da idade, o esperado era encontrarmos correlações negativas, pois geralmente acredita-se que uma menor idade para o início da aprendizagem está associada a uma produção em L2 ou L3 mais próxima do padrão nativo, nesse caso, um maior valor de VOT ( $c f$. BIRDSONG, 2006). Contudo, as correlações significativas verificados para as variáveis relacionadas à idade são todas positivas, inclusive no que se refere às produções em PB. É possível sugerir, dessa maneira, que até um aprendiz tardio de uma língua adicional pode realizar produções em L2 ou L3 semelhantemente aos nativos, reforçando a contestação à ideia de um período crítico para aprendizagem de língua estrangeira (previamente contestado por Flege, 1995). Além disso, também é possível argumentar, a partir dos dados, que as L2 e L3 estão bastante imbricadas, e que considerar uma variável referente ao início de estudo de apenas uma das línguas não é suficiente, provendo mais evidências para a característica de não-linearidade prevista em SDC.

Por fim, seguindo Lima Jr. (2016) e a própria concepção de língua como SDC, que considera a variabilidade como essencial para a mudança, é apresentada, aqui, uma análise qualitativa mais individual desses dados obtidos pelos participantes multilíngues, pois a literatura descrita na Introdução deste artigo aponta que nem sempre os resultados de um grupo refletem as trajetórias individuais. Primeiramente, ao se olhar somente para as produções individuais do Grupo A, dos 10 participantes bilíngues (descritos na Tabela 1), podemos ver que: (i) para /p/, sete participantes bilíngues apresentam uma média de valores de VOT no PB maiores do que o maior valor produzido por um monolíngue $(25,09 \mathrm{~ms})^{67}$; (ii) para / $t$ /, três participantes bilíngues apresentam valores de VOT no PB maiores do que o maior valor produzido por um monolíngue $(31,44 \mathrm{~ms})$, inclusive o participante que começou a

\footnotetext{
${ }^{67}$ Reiteramos que os dados para controle, referentes aos monolíngues, são extraídos de Kupske (2016).
} 
aprender Inglês mais cedo (Participante 2 - 1 ano de idade); e (iii) para /k/, também são três os participantes bilíngues que apresentam valores de VOT no PB maiores do que o maior valor produzido por um monolíngue $(65,86 \mathrm{~ms})$, inclusive o segundo participante que começou a aprender Inglês mais cedo (Participante 9 - 3 anos).

No entanto, ao se olhar somente para o Grupo B, dos 10 participantes trilíngues, é possível ver que: (i) para /p/, cinco participantes trilíngues apresentam uma média de valores de VOT no PB maiores do que o maior valor produzido por um monolíngue (25,09 ms); (ii) para / $t$ /, seis participantes trilíngues apresentam uma média de valores de VOT no PB maiores do que o maior valor produzido por um monolíngue (31,44 ms); e (iii) para $/ \mathrm{k} /$, novamente, são três os participantes bilíngues que apresentam valores de VOT no PB maiores do que o maior valor produzido por um monolíngue $(65,86 \mathrm{~ms})$. Desses três últimos, pode-se chamar a atenção para (i) o Participante 20, que é o participante mais velho (54 anos de idade), com a maior experiência em Inglês (42 anos de estudo), e que produziu o valor mais alto de VOT em PB (78, $21 \mathrm{~ms})$; (ii) o Participante 16, que tem a maior soma do número de horas de uso em Inglês (10,5 horas/dia), e começou a aprender e a utilizar Alemão mais cedo (10 e 14 anos de idade, respectivamente); e (iii) o Participante 14, que tem a maior soma do número de horas de uso de Alemão (3,6 horas/dia).

\section{Considerações Finais}

De maneira geral, é possível dizer que a alta variabilidade dos dados individuais de histórico linguístico não possibilitou correlações muito claras ou estatisticamente significativas, apontando realmente para a necessidade de um olhar mais individual para esses dados, como previsto por Lowie e Verspoor (2019) dentro da concepção de língua como SDC, olhar esse que levou a um entendimento maior sobre os inúmeros fenômenos em ocorrência no sistema híbrido desses participantes multilíngues. Ainda dentro das discussões inicialmente propostas por este estudo, pode-se concluir, com base nos resultados encontrados, que há uma clara relação entre todas as línguas dos sistemas desses participantes, pois foram encontradas correlações significativas entre as línguas em evidência. Embora com resultados diferenciados entre os grupos, o grupo de bilíngues mostrou-se como uma boa amostra de dados para a discussão sobre atrito linguístico, pois, pelos resultados, há correlações positivas entre o VOT da L1 e da L2. Já no caso dos trilíngues, não foram verificadas correlações entre o VOT da L1 e das L2 e L3, mas sim entre as duas últimas, o que configura o grupo como uma boa amostra para a discussão da influência mútua entre as línguas adicionais. Ainda em consonância com a concepção de língua como SDC, foi possível observar, também, que as variáveis particulares de uso de língua por cada grupo de participantes acarretam diversas alterações no sistema. Tal fato deixa claro que são múltiplos os agentes em interação o tempo todo, causando a mudança no sistema fonético-fonológico desses falantes.

\section{Referências}

ABRAMSON, A.; WHALEN, D. Voice Onset Time (VOT) at 50: theoretical and practical issues in measuring voicing distinctions. Journal of Phonetics, v. 63, p. 75-86, 2017.

BECKNER, C.; ELLIS, N.; BLYTHE, R.; HOLLAND, J.; BYBEE, J.; KE, J.; CHRISTIANSEN, M.; LARSEN-FREEMAN, D.; CROFT, W.; SCHOENEMANN, T. Language is a Complex Adaptive System - Position Paper. Language Learning, v. 59, supl. 1, p. 1-26, 2009. 
BEST, C. A direct realist view of cross-language speech perception. In: STRANGE, W. (ed.). Speech Perception and Linguistic Experience: theoretical and methodological issues in cross-language speech research. Timonium: York Press, 1995, p. 167-200.

; TYLER, M. Nonnative and second-language speech perception: commonalities and complementarities. In: BOHN, O.; MUNRO, M. Language Experience in Second Language Speech Learning: In honor of James Emil Flege. Amsterdam/Philadelphia: John Benjamins Publishing Company, 2007, p. 13-34.

BOERSMA, P.; WEENINK, D. Praat: doing phonetics by computer - version 6.0.40. 2018. Disponível em www.praat.org.

DE BOT, K. Epilogue. In: VERSPOOR, M.; de BOT, K.; LOWIE, W. (eds.). A Dynamic Approach to Second Language Development: methods and techniques. Amsterdam: John Benjamins Publishing Company, 2011, p. 123-127.

DE BOT, K.; Complexity Theory and Dynamic Systems Theory: same or different? In: ORTEGA, L.; HAN, Z.H. (eds.). Complexity Theory and Language Development: in celebration of Diane Larsen- Freeman. Amsterdam: John Benjamins Publishing Company, 2017, p. 51-58.

; LOWIE, W.; VERSPOOR, M. A Dynamic Systems Theory approach to second language acquisition. Bilingualism: Language \& Cognition, p. 7-21, 2007.

; THORNE, S.; VERSPOOR, M. Dynamic Systems Theory as a comprehensive theory of second language development. In: MAYO, M.; MANGADO, M.; ADRIÁN, M. (eds.). Contemporary Approaches to Second Language Acquisition. John Benjamins Publishing Company, 2013, p. 199-220.

DE LEEUW, E. How phonetics and phonology inform L1 attrition (narrowly defined) research. Linguistic Approaches to Bilingualism 7:6, p. 725-729, 2017.

Phonetic Attrition. In: SCHMID, M. S; KÖPKE, B. (Eds.) Handbook of Attrition. Oxford: Oxford University Press, 2018, p. 1-32.

; MENNEN, I.; SCOBBIE, J. Dynamic systems, maturational constraints and L1 phonetic attrition. International Journal of Bilingualism, v. 17, n. 6, p. 683-700, 2012.

FINGER, Ingrid; ORTIZ-PREUSS, Elena. A psicolinguística do bilinguismo: estudando o processamento linguístico e cognitivo bilíngue. In: ORTIZ-PREUSS, E.; FINGER, I. (orgs). A dinâmica do processamento bilíngue. Campinas: Pontes Editores, 2018, p. 31-57.

FLEGE, James E. The production of "new" and "similar" phones in a foreign language: Evidence for the effect of equivalence classification. Journal of Phonetics, 15, p. 47-65, 1987.

E. Second Language Speech Learning: Theory, Findings, and Problems. In: STRANGE, W. (ed.) Speech perception and linguistic experience: issues in cross language research. Timonium, MD: York press, 1995, p. 233-277.

Assessing constraints on second-language segmental production and perception. In: MEYER, A.; SCHILlER, N. (eds). Phonetics and Phonology in Language Comprehension and Production, Differences and Similarities. Berlin: Mouton de Gruyter, 2003, p. 319-355.

Language contact in bilingualism: Phonetic system interactions. In: COLE, J.; HUALDE. J. (Eds.), Laboratory phonology, 9. Berlin, Ger.: Walter de Gruyter, 2007, p. 353382.

GROSJEAN, F. Neurolinguists, beware! The bilingual is not two monolinguals in one person. Brain and Language, 1989, 36(1), p. 3-15, 1989.

HERDINA, P.; JESSNER, U. A Dynamical Model of Multilingualism: Perspectives of Change in Psycholinguistics. Cromwell Press Ltd., 2002.

KLEIN-BRALEY, C.; RAATZ, U. Introduction to language testing and C-tests. In: J.A. COLEMAN, R. GROTJAHN (Eds.). University language testing and the C-test, p. 75-91, 2002. 
KUPSKE, F. F. Imigração, Atrito e Complexidade: a produção das oclusivas surdas iniciais do Inglês e do Português por Sul-Brasileiros residentes em Londres. Tese (Doutorado em Letras). Porto Alegre: Universidade Federal do Rio Grande do Sul, 2016.

LARSEN-FrEEMAN, D.; CAMERON, L. Complex Systems and Applied Linguistics. Oxford University Press, 2008.

LIMA JR., R. M. A necessidade de dados individuais e longitudinais para análise do desenvolvimento fonológico de L2 como sistema complexo. Revista Virtual de Estudos da Linguagem, v. 14, n. 27, p. 203-225, 2016.

LISKER, L.; ABRAMSON, A. A cross-language study of voicing in initial stops: acoustical measurements. Word, v. 20, p. 384-422, 1964.

LOWIE, W. Lost in state space? Methodological considerations in Complex Dynamic Theory approaches to second language development research. In: ORTEGA, L.; HAN, Z.H. (eds.). Complexity Theory and Language Development: in celebration of Diane LarsenFreeman. Amsterdam: John Benjamins Publishing Company, 2017, p. 123-141.

; VERSPOOR, M. Variability and variation in Second Language Acquisition orders: a dynamic reevaluation. Language Learning, v. 65, n. 1, p. 63-88, 2015.

$\overline{69, \text { S.1 }}, \overline{\text { p. } 184}-206,2019$.

Individual differences and the ergodicity problem. Language Learning, v.

PARK, E. S. Language Attrition. In: LIONTAS, John I (Ed.) The TESOL Encyclopedia of English Language Teaching. John Wiley \& Sons, p. 1-12, 2018.

PEREYRON, L. A produção vocálica por falantes de Espanhol (L1), Inglês (L2) e Português (L3): uma perspectiva dinâmica na (multi) direcionalidade da transferência linguística. Tese (Doutorado em Letras). Porto Alegre: Universidade Federal do Rio Grande do Sul, 2017.

PEROZZO, R. V. Sobre as esferas cognitiva, acústico-articulatória e realista indireta da percepção fônica não-nativa: para além do PAM-L2. Tese (Doutorado em Letras). Porto Alegre: Universidade Federal do Rio Grande do Sul, 2017.

SCHERESCHEWSKY, Laura Castilhos. O atrito linguístico sobre os padrões de VOT do Português: efeitos do Inglês (L2) e do Alemão (L3) em aprendizes bilíngues e trilíngues. Trabalho de Conclusão de Curso (Graduação em Letras) - Universidade Federal do Rio Grande do Sul, Porto Alegre, 2018.

SCHMID, M.; DE LEEUW, E. Section III Introduction 16. Linguistic factors. In: The Oxford Handbook of Language Attrition. Oxford: Oxford University Press, 2018.

; KOPKE, B. The relevance of first language attrition to theories of bilingual development. Linguistic Approaches to Bilingualism, v. 7, n. 6, p. 638-667, 2017.

SCHOLL, A. P. Proficiência autoavaliada através de um questionário de histórico da linguagem. Dissertação (Mestrado em Letras). PPG Letras - Universidade Federal do Rio Grande do Sul, Porto Alegre, RS, 2016.

; FINGER, I. Elaboração de um Questionário de Histórico da Linguagem para pesquisas com bilíngues. Nonada: Letras em revista, v. 2, p. 1-17, 2013.

SURRAIN, S.; LUK, G. Describing bilinguals: A systematic review of labels and descriptions used in the literature between 2005-2015. Bilingualism: Language and Cognition, 2017.

Recebido em 20/06/2019

Aceito em 02/08/2019 